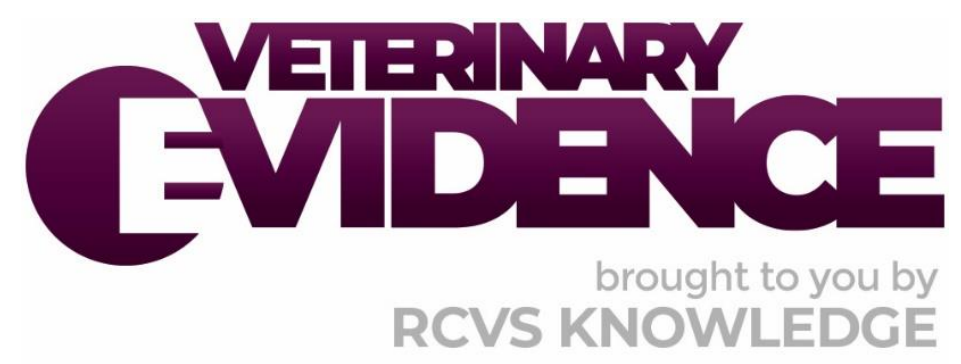

\title{
Should we offer total hip replacement to our feline patients?
}

A Knowledge Summary by

Katie Smithers BVSC CertAVP MRCVS ${ }^{1^{*}}$

\footnotetext{
${ }^{1}$ University of Liverpool, Leahurst Campus, Neston, CH64 7TE

*Corresponding Author (kasmithers789@outlook.com)
}

ISSN: 2396-9776

Published: 16 Dec 2020

in: The Veterinary Evidence journal Vol 5, Issue 4

DOI: $10.18849 /$ VE.V5I4.347

Reviewed by: Jacqueline Cole (BSc BVetMed MRCVS) and Eithne Comerford (MVB PhD CertVR CertSAS PGCertHE DipECVS FHEA FRCVS RCVS)

Next Review Date: 11 Jan 2022 


\section{KNOWLEDGE SUMMARY}

\section{PICO question}

In cats with traumatic coxofemoral injury, does total hip replacement (THR) offer improved outcome when compared with femoral head and neck excision (FHNE) arthroplasty?

\section{Clinical bottom line}

\section{Category of research question}

\section{Treatment}

\section{The number and type of study designs reviewed}

One paper was critically reviewed. It was a non-randomised retrospective observational study

\section{Strength of evidence}

Moderate evidence

\section{Outcomes reported}

THR results in superior clinical outcome and owner satisfaction compared to FHNE in cats

\section{Conclusion}

In cats with traumatic coxofemoral injury, although the evidence is not conclusive and somewhat limited, the literature reviewed here suggests that THR offers a superior outcome in feline patients.

There is currently insufficient evidence to determine if there is a difference in long-term outcome, complications or osteoarthritis (OA) development following THR or FHNE in feline patients

\section{How to apply this evidence in practice}

The application of evidence into practice should take into account multiple factors, not limited to: individual clinical expertise, patient's circumstances and owners' values, country, location or clinic where you work, the individual case in front of you, the availability of therapies and resources.

Knowledge Summaries are a resource to help reinforce or inform decision making. They do not override the responsibility or judgement of the practitioner to do what is best for the animal in their care.

\section{Clinical Scenario}

A 5-year-old male neutered cat, with a bodyweight of $5.1 \mathrm{~kg}$ presented with an acute history of unilateral pelvic limb lameness. On clinical examination pain was localised to the coxofemoral (hip) joint. Orthogonal radiography of the affected joint demonstrated the presence of craniodorsal coxofemoral luxation.

Surgical treatment options were discussed with the client. Both total hip replacement (THR) and femoral head and neck excision arthroplasty (FHNE) are performed locally; at referral centres and with the practice respectively and are financially feasible.

Therefore the applicable evidence based question was: when considering postoperative affected pelvic limb function, owner satisfaction and complication rates, what evidence is there to suggest a THR would provide a superior outcome to FHNE? 


\section{The evidence}

Only a single non-randomised retrospective observational study was identified as relevant to the question and as such the evidence base, with which to answer the question, is low.

Abbreviations:

THR - Total hip replacement

FHNE - Femoral head and neck excision

OA - Osteoarthritis

\section{Summary of the evidence}

\begin{tabular}{|c|c|}
\hline 09) & \\
\hline Population: & $\begin{array}{l}\text { Feline patients with capital epiphyseal fracture or coxofemoral } \\
\text { luxation of less than } 10 \text { days duration }\end{array}$ \\
\hline Sample size: & Eight cats \\
\hline Intervention details: & $\begin{array}{l}\text { THR ( } \mathrm{n}=3 \text { cats); } \\
\text { - Cranial lateral approach (partial deep gluteal tenectomy) } \\
\text { or femoral head ostectomy ( } \mathrm{n}=5 \text { cats); } \\
\text { - FHNE } \\
\text { Thigh circumference }(\mathrm{cm}) \text { to assess muscle mass, hip flexion } \\
\text { (degrees), hip extension (degrees) and dorsal femoral displacement } \\
\text { (mm) were measured in both limbs for cats undergoing either } \\
\text { procedure }\end{array}$ \\
\hline Study design: & Case-control study (retrospective, observational, non-randomised) \\
\hline Outcome studied: & $\begin{array}{l}\text { Objective measurements: } \\
\text { - Thigh circumference }(\mathrm{cm}) \text { to assess muscle mass } \\
\text { - Hip flexion (degrees) } \\
\text { - Hip extension (degrees) } \\
\text { All the above were measured in the operated and non-operated } \\
\text { limb } \\
\text { Subjective measurements: } \\
\text { - Pain upon joint range of motion measured via goniometry } \\
\text { - Gait evaluation - as assessed by the veterinary surgeon at } \\
\text { - } \text { follow-up appointments } \\
\text { Owner assessment - done via owner interview at the time of } \\
\text { last visit }\end{array}$ \\
\hline $\begin{array}{l}\text { Main findings: } \\
\text { (relevant to PICO question): }\end{array}$ & $\begin{array}{l}\text { - Superior hip extension (degrees) with THR } 148^{\circ} \pm 1^{\circ}(98 \%) \\
\text { compared with FHNE } 138^{\circ} \pm 8^{\circ}(96 \%) \\
\text { - No dorsal femoral displacement with THR, compared with } \\
\text { approximately } 11 \mathrm{~mm} \text { displacement in FHNE group } \\
\text { (measured by comparing the distance from the centre of the } \\
\text { dorsal aspect of the acetabular rim to the proximal aspect of } \\
\text { the greater trochanter on the operated and non-operated } \\
\text { side on a lateral radiograph with the hemipelves } \\
\text { superimposed) } \\
\text { Superior return to normal thigh circumference following THR } \\
\text { compared to FHNE ( } 98 \% \text { compared with } 92 \%)\end{array}$ \\
\hline
\end{tabular}




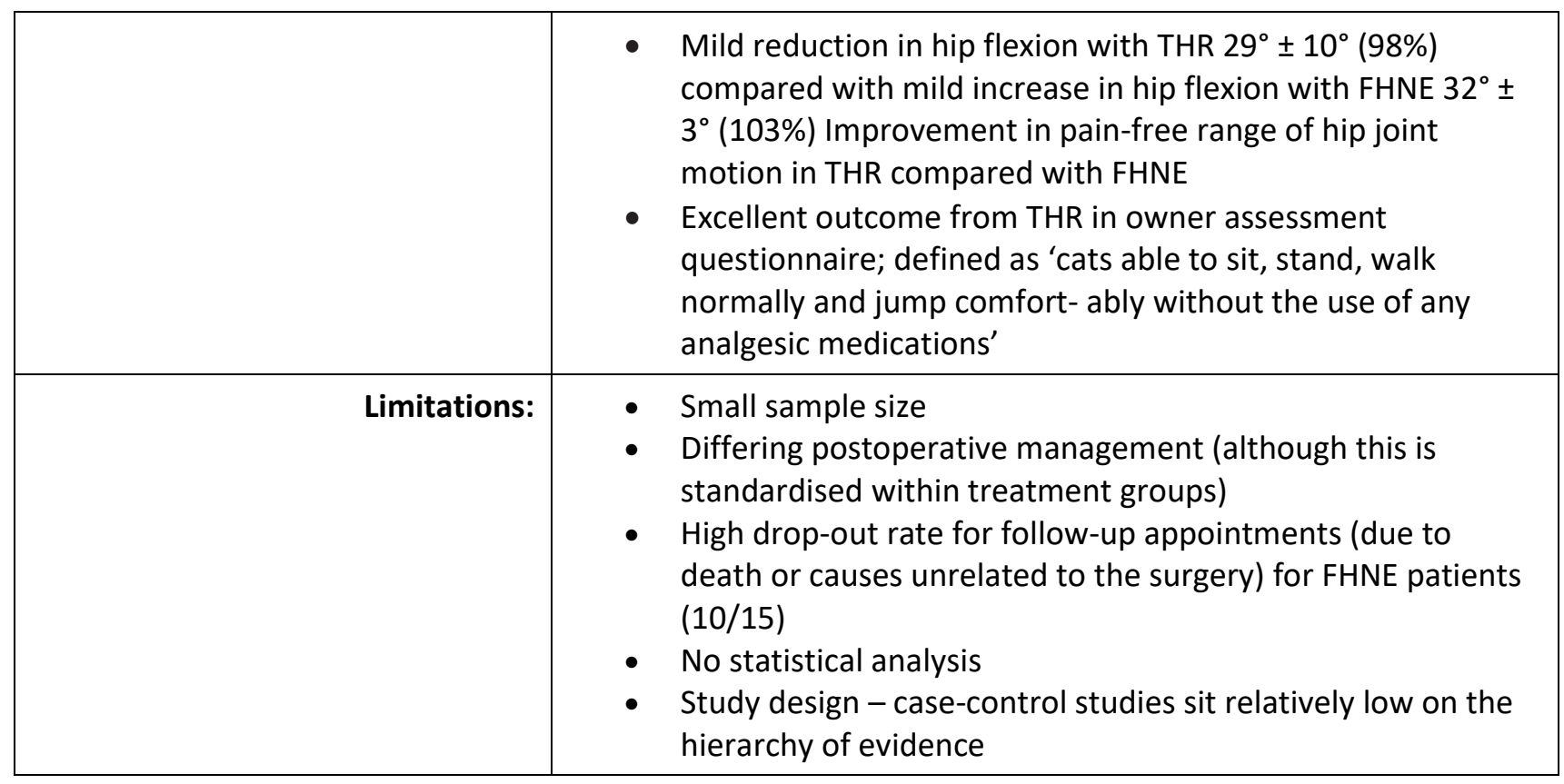

\section{Appraisal, application and reflection}

Only one paper was identified as directly relating to the question, and was a non-randomised retrospective observational study; which is a type of case-control study and is therefore low on the evidence pyramid. Therefore, both meta-analysis and randomised control trials would provide a superior level of evidence but have not yet been carried out in relation to this PICO question.

The study identified focused on objective and subjective measurements for both functional outcomes and owner satisfaction following THR when compared with FHNE surgical procedures. In this study the authors, Liska et al. (2009), reported superior recovery from THR when compared with FHNE based on the clinical assessment of muscle mass, hip passive range of motion, gait analysis and owner assessment. Dorsal femoral displacement was also absent from the THR group postoperatively. This demonstrates a superior outcome for the THR group using both subjective and objective measurements.

The study advises further studies should be carried out with a blinded randomised controlled trial being most appropriate to provide an evidence-based answer to this PICO question.

THR has been reported to be successful in $41 / 49$ (83\%) cases in equivalent canine populations; such as small breed dogs undergoing micro THR (Liska, 2010). In addition, the findings regarding inferior FHNE outcome are in agreement with similar findings in both large and small breed dogs undergoing this procedure (Warnock et al., 2003).

Complication rates within a population would have to be extrapolated from studies in other species. For example, aseptic loosening was seen in only six $(0.7 \%)$ of cases in a study of 964 people 24 years after surgery (Cameron, 2008) and in dogs a total complication rate of 25/306 (9\%) cases was noted in a recent multicentre registry study of THR outcomes in canine patients (Henderson et al., 2017) and a femoral fracture rate of 16/684 THR (Liska, 2004).

Rehabilitation and postoperative management present a more complicated picture in cats and may also affect clinical outcome and owner decision making, this study recommends cage rest which may not be possible in many feline patients following feline THR. Therefore, it is important to be able to make comparison in outcome between both procedures with the best level of evidence possible. This leads to the need to consider papers 
reporting positive functional outcome following THR in cats, despite the fact these are non-comparative, due to the minimal available evidence applicable to the question. Multiple studies have reported satisfactory to excellent medium to long-term outcomes with FHNE in feline patients providing this procedure is adequately performed (Yap et al., 2014; and Off \& Matis, 2010).

Therefore, the conclusions made within the clinical bottom line component of this evidence based Knowledge Summary are based on the following points:

- The best quality paper available to answer the proposed question reported an improvement in thigh muscle mass and measured passive range of motion in flexion and extension as well as a reduction in pain upon palpation following THR when compared with FHNE carried out by the same board certified specialist surgeon.

- This paper also looked at owner satisfaction as an outcome for each procedure and found a reported improvement in the THR group compared to the FHNE group.

- There was no data provided with this study on complication rates for both procedures.

Limitations on extrapolating the data reported here to make recommendations for changes in current clinical practice are as follows:

- All procedures reported were performed by the same board certified specialist surgeon at one referral centre. This assumes this data can be extrapolated to THR or FHNE performed in a first-opinion practice or by surgeons with limited experience of one particular procedure, which may not be applicable.

- This evidence based medicine (EBM) search only identified one paper which, although applicable to answering the question, is limited not only by study design but also by sample size and lack of statistical analysis, despite both objective and subjective measures of outcome being utilised within its design.

- This summary as above assesses the impact of procedure selection on a limited number of measured predominantly clinical outcomes. Other factors such as cost, hospitalisation, aftercare and rehabilitation requirements are not considered and are all factors likely to influence client decision making.

- It is also worth noting that THR and FHNE may be considered for developmental orthopaedic disease such as hip dysplasia and although this paper is suggestive of a superior short-term outcome from THR this may not be the case in patients requiring surgery at a younger age or following development of osteoarthritis. Therefore, further studies in this area would be indicated before data can be extrapolated to allow decision making on all cases where THR or FHNE are the potential surgical treatment options available in clinical practice.

In conclusion this suggests that THR may result in superior outcome and owner satisfaction when compared to FHNE however further research including larger sample size, multi-centre, randomised, controlled clinical trials and more objective determination of the outcomes of surgery using more objective measurements such as client metrology instruments (Stadig et al., 2019) and pressure platform analysis would be indicated. 


\section{Methodology Section}

\begin{tabular}{|r|l|}
\hline \multicolumn{1}{|l|}{ Search Strategy } \\
$\begin{aligned} \text { Databases searched and dates } \\
\text { covered: }\end{aligned}$ & $\begin{array}{l}\text { 1. CAB Abstracts on OVID Platform (1973-2019) } \\
\text { 2. PubMed accessed via the NCBI website (1910-2019) }\end{array}$ \\
\hline Search terms: & $\begin{array}{l}\text { CAB Abstracts: } \\
\text { (feline OR felines OR cat OR cats) AND ((total AND hip AND } \\
\text { replacement) OR THR) AND (osteotomy OR excision OR arthroplasty OR } \\
\text { FHNE }\end{array}$ \\
& $\begin{array}{l}\text { PubMed: } \\
\text { (feline OR felines OR cat OR cats) AND ((total AND hip AND } \\
\text { replacement) OR THR) AND (osteotomy OR excision OR arthroplasty OR } \\
\text { FHNE }\end{array}$ \\
\hline Dates searches performed: & \begin{tabular}{l} 
11 Jan 2020 \\
\hline
\end{tabular} \\
\hline
\end{tabular}

\section{Exclusion / Inclusion Criteria}

\begin{tabular}{|c|c|}
\hline Exclusion: & $\begin{array}{l}\text { - } \text { Case reports } \\
\text { - } \text { Experimental papers } \\
\text { - } \text { Duplicates } \\
\text { - Non-comparative papers } \\
\text { - Non-English language papers }\end{array}$ \\
\hline Inclusion: & $\begin{array}{l}\text { - } \text { English language } \\
\text { - } \text { Peer reviewed publication } \\
\text { - } \text { Original data } \\
\text { - In vivo study } \\
\text { - } \quad \text { Feline patients } \\
\text { - } \text { Comparative papers including both THR and FHNE }\end{array}$ \\
\hline
\end{tabular}

\begin{tabular}{|c|c|c|c|c|c|c|c|}
\hline Database & $\begin{array}{l}\text { Number } \\
\text { of } \\
\text { results }\end{array}$ & $\begin{array}{l}\text { Excluded } \\
\text { - non- } \\
\text { feline } \\
\text { patients }\end{array}$ & $\begin{array}{l}\text { Excluded - } \\
\text { case } \\
\text { reports }\end{array}$ & $\begin{array}{l}\text { Excluded - } \\
\text { non-English } \\
\text { language }\end{array}$ & $\begin{array}{l}\text { Excluded - } \\
\text { did not } \\
\text { include both } \\
\text { THR and } \\
\text { FHNE }\end{array}$ & $\begin{array}{l}\text { Excluded - } \\
\text { non- } \\
\text { comparative }\end{array}$ & $\begin{array}{c}\text { Total } \\
\text { relevant } \\
\text { papers }\end{array}$ \\
\hline $\begin{array}{l}\mathrm{CAB} \\
\text { Abstracts }\end{array}$ & 12 & 1 & 3 & 0 & 1 & 7 & 0 \\
\hline PubMed & 40 & 9 & 4 & 11 & 8 & 7 & 1 \\
\hline
\end{tabular}


The author declares no conflicts of interest.

\section{REFERENCES}

1. Cameron, H.U. (2008). Cemented Femoral Fixation: Thin Mantles, The French Paradox. Seminars in Arthroplasty.19(1), 144-147. DOI: http://dx.doi.org/10.1053/i.sart.2007.12.018

2. Henderson, E.R., Wills, A., Torrington, A.M., Moores, A.P., Thomson, D., Arthurs, G., Brown, G., Denny, H.R., Scott, H.W., MacQueen, I., Dunne, J., Onyett, J., Walker, J.D., Prior, J., Owen, M.R., Burton, N., Whitelock, R., Girling, S., Morrison, S., Gilbert, S., Langley-Hobbs, S.J., Gemmill, T.J., Störk, C.K., Bright, S., Comerford, E., Pettitt, R., Macdonald, N. and Innes, J.F. (2017). Evaluation of variables influencing success and complication rates in canine total hip replacement: results from the British Veterinary Orthopaedic Association Canine Hip Registry (collation of data: 2010-2012). Veterinary Record. 181(1), 18. DOI: http://dx.doi.org/10.1136/vr.104036

3. Liska, W.D. (2004). Femur fractures associated with canine total hip replacement. Veterinary Surgery. 33(2), 164-172. DOI: http://dx.doi.org/10.1111/j.1532-950X.2004.04024.x

4. Liska, W.D. (2010). Micro total hip replacement for dogs and cats: surgical technique and outcomes. Veterinary Surgery. 39(7), 797-810. DOI: http://dx.doi.org/10.1111/i.1532950X.2010.00725.x

5. Liska, W.D., Doyle, N., Marcellin-Little, D.J. and Osborne, J.A. (2009). Total hip replacement in three cats: surgical technique, short-term outcome and comparison to femoral head ostectomy. Veterinary and Comparative Orthopaedics and Traumatology. 22(6), 505-510.

DOI: http://dx.doi.org/10.3415/VCOT-08-09-0087

6. Off, W. and Matis U. (2010). Excision arthroplasty of the hip joint in dogs and cats. Clinical, radiographic, and gait analysis findings from the Department of Surgery. Veterinary Faculty of the Ludwig-Maximi- lians-University of Munich, Germany. Veterinary and Comparative Orthopaedics and Traumatology. 23, 297-305.

7. Stadig, S., Lascelles, B.D.X., Nyman, G. and Bergh, A. (2019). Evaluation and comparison of pain questionnaires for clinical screening of osteoarthritis in cats. The Veterinary Record. 185(24), 757. DOI: http://dx.doi.org/10.1136/vr.105115

8. Warnock, J.J., Dyce, J., Pooya, H. and Schulz, K.S. (2003). Retrospective analysis of canine miniature total hip prostheses. Veterinary Surgery. 32(3), 285-291.

DOI: http://dx.doi.org/10.1053/jvet.2003.50034

9. Yap, F.W., Dunn, A.L., Garcia-Fernandez, P.M., Brown, G., Allan, R.M. and Calvo, I. (2014). Femoral head and neck excision in cats: medium-to long-term functional outcome in 18 cats. Journal of Feline Medicine and Surgery. 17(8), 704-710. DOI: http://dx.doi.org/10.1177/1098612X14556848 


\section{EVIIDEFeE

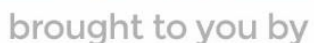 \\ RCVS KNOWLEDGE}

\section{Intellectual Property Rights}

Authors of Knowledge Summaries submitted to RCVS Knowledge for publication will retain copyright in their work, and will be required to grant RCVS Knowledge a non-exclusive license of the rights of copyright in the materials including but not limited to the right to publish, re-

publish, transmit, sell, distribute and otherwise use the materials in all languages and all media throughout the world, and to license or permit others to do so.

\section{Disclaimer}

Knowledge Summaries are a peer-reviewed article type which aims to answer a clinical question based on the best available current evidence. It does not override the responsibility

of the practitioner. Informed decisions should be made by considering such factors as individual clinical expertise and judgement along with patient's circumstances and owners' values. Knowledge Summaries are a resource to help inform and any opinions expressed within the Knowledge Summaries are the author's own and do not necessarily reflect the view of the RCVS Knowledge. Authors are responsible for the accuracy of the content. While the

Editor and Publisher believe that all content herein are in accord with current recommendations and practice at the time of publication, they accept no legal responsibility

for any errors or omissions, and make no warranty, express or implied, with respect to material contained within.

For further information please refer to our Terms of Use.

RCVS Knowledge is the independent charity associated with the Royal College of Veterinary Surgeons (RCVS). Our ambition is to become a global intermediary for evidence based veterinary knowledge by providing access to information

that is of immediate value to practicing veterinary professionals and directly contributes to evidence based clinical decision-making.

https://www.veterinaryevidence.org/

RCVS Knowledge is a registered Charity No. 230886.

Registered as a Company limited by guarantee in England and Wales No. 598443.

Registered Office: Belgravia House, 62-64 Horseferry Road, London SW1P 2AF



This work is licensed under a Creative Commons Attribution 4.0 International License 